\title{
Use of Filled Bioplastics in Construction
}

\author{
Ruslan Safin ${ }^{1 *[0000-0002-0226-4232]}$, Nour Galyavetdinov ${ }^{1}$, Regina Salimgaraeva ${ }^{1}$, Guzel Ilalova ${ }^{1}$, \\ and Ksenia Saerova ${ }^{1}$
}

${ }^{1}$ Kazan National Research Technological University, 420015 Kazan, Russia

\begin{abstract}
Polymers and associated composite materials play an increasingly prominent role among structural materials. The relevance of the use of bioplastics as an alternative to synthetic polymers increases year by year. In this regard, the paper describes the production of a ligno-filled polymer material based on polyhydroxyalkanoates to be used in the production of building materials. The studies allowed us to produce the material from wood hydrolyzates. The highest yield of reducing substances in course of pine sawdust hydrolysis with $4 \%$ sulfurous acid occurred at $170^{\circ} \mathrm{C}$. To obtain a biopolymer in liquid wood hydrolyzate medium, the Cupriavidus necator strain was used, which after 50 hours of cultivation gave a biopolymer yield of up to $15 \mathrm{~g} / \mathrm{l}$. The studies of the strength characteristics of the composite based on the obtained bioplastic and dried solid wood hydrolysates allowed us to produce a balanced composition for subsequent use in construction.
\end{abstract}

Keywords. Wood, hydrolysis, polyhydroxyalkanoates, biopolymers, composite.

\section{Introduction}

In recent decades, polymer building materials have become widely used in finishing work. However, using naturally non-destructible petrochemical plastics poses the problem of their disposal at the end of the life cycle, thereby increasing the dangerous environmental impacts. Therefore, the problem of creating building materials based on biodegradable polymers is relevant today. Such polymers include polyhydroxyalkanoates (PHA), a family of polyesters that are naturally synthesized by microorganisms. The resulting polymers allow producing materials with various characteristics ranging from hard to extremely elastic. Among those produced from renewable resources, the most promising now are PHAs, a family of polymers with a wide range of properties that can in many cases exceed the performance achieved using synthetic polymers.

The crucial properties of polyhydroxyalkanoates are biocompatibility and biodegradability. Biodegradation is a process of changes in the chemical composition of a substance caused by microorganisms, which results in the formation of natural end metabolites. PHAs are decomposed by microorganisms in an active microbiological environment (i.e., in soil, fresh water, seawater, compost, etc.), even under anaerobic conditions, but are stable in clean water. They are also biocompatible. This means that they

${ }^{*}$ Corresponding author: cfaby@mail.ru 
are non-toxic and do not cause any of the known allergic reactions. Thanks to such properties, PHAs are of great interest as a raw material to produce finishing building materials.

Polyhydroxyalkanoates (PHAs) can be produced from various substrates, including individual compounds (carbon dioxide and hydrogen, sugars, alcohols, organic acids); alcoholic, sugar and hydrolysis industries, olive and palm oil production waste. These studies are described in $[1,2]$. In addition, non-traditional substrates, including toxic ones, are also used. It was found that PHA can be synthesized from poorly soluble and toxic octane and octanoate, sodium benzoate, phenol, and methacrylic acid [3-5].

Over the past few years, many studies have been focused on PHA production based on industrial by-products and waste, such as agricultural raw materials, used vegetable oils, or waste water. The use of inexpensive waste carbon sources can reduce the cost of producing PHAs and increase their sustainability $[6,7]$.

A nearly inexhaustible source of raw materials for large-scale PHA production is plant biomass formed in huge quantities annually. Waste from various crops containing polysaccharides with various structures and compositions can be hydrolyzed to produce a range of water-soluble sugars.

Hydrolysis industry comprises a number of industries. Their technology is based on the hydrolytic destruction of plant materials [8-14]. This process is carried out by the catalytic conversion of natural wood polysaccharides (cellulose and hemicellulose) into monosaccharides, which are then used to produce a number of high-value products [15-19]. The content of polysaccharides in plant materials reaches $55-75 \%$.

Most of the existing hydrolysis plants are alcohol and yeast production facilities where ethyl alcohol is produced from hexose sugars and feed yeast is produced from pentose sugars. Vegetable raw materials are then subjected to percolation hydrolysis in the presence of diluted sulfuric acid. The theoretical sugar recovery reaches $65-70 \%$. In order to increase the efficiency of using plant raw materials and create a waste-free technology, it is advisable to use both the sugars isolated during hydrolysis, and the solid residue [20-25].

Therefore, a promising area of research is the development of PHA from wood hydrolyzates, as well as a comprehensive technology for producing a composite material based on polyhydroxyalkanoate and dried solid residue after wood hydrolysis. This composite can be widely used as siding for building facades and other facing products for construction.

\section{Materials and methods}

For hydrolysis, sawdust of Scots pine (Pinus Sylvestris L.) with a fraction of up to $2 \mathrm{~mm}$ and laboratory sulfurous acid were used as raw materials.

An important parameter in hydrolysis is the hydromodulus (the ratio of the vegetable raw material weight to the weight or volume of an aqueous acid solution) [21]. Depending on the hydromodulus value, the yield of sugar from the raw material, its concentration in the hydrolysate, and the acid consumption significantly change. The hydromodulus of 1:5.8 was selected experimentally.

Acid hydrolysis was carried out on a hydrolyser with a heat accumulator, consisting of six sealed stainless $30 \mathrm{ml}$ capsules (Fig. 1). Sawdust previously weighed on analytical scales, was placed in capsules using a funnel with a subsequent addition of calculated amounts of distilled water and sulfurous acid under a draught. Further, the capsules containing the study object were simultaneously placed in a hydrolyser preheated to the test temperature. 


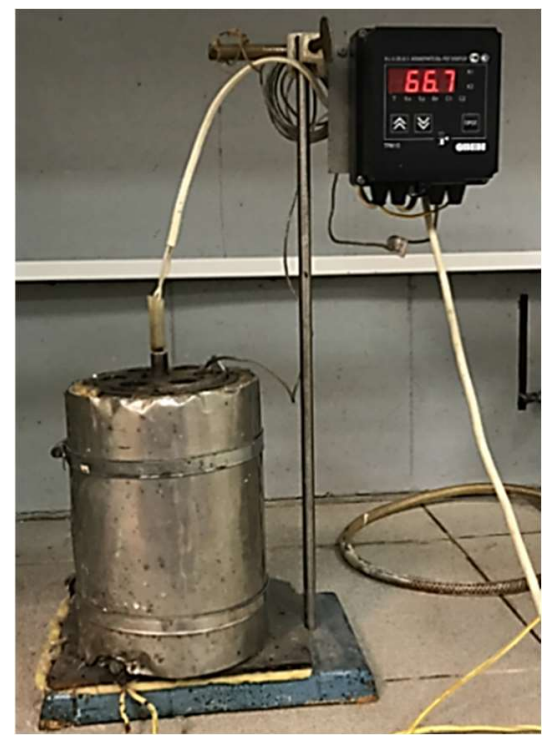

Fig. 1. Capsule type hydrolyser with a metal heat accumulator.

Hydrolysis proceeded as follows: the capsules were sequentially removed from the hydrolyzing unit at a certain time interval $(20,40,60,80,100,120$ minutes for a temperature of $160^{\circ} \mathrm{C}$ and $10,20,30,40,60,90$ minutes for a temperature of $170^{\circ} \mathrm{C}$ ) using a special gripper and cooled with running water. After the hydrolysis, the contents of the capsules were separated by straining into the liquid and dry residue to further determine the content of reducing substances.

The amount of reducing substances was determined using the Macaigne-Schoorl method, which consists in using a Fehling solution with a given copper content and iodometric determination of the amount of copper (II) unspent during the reaction. The separated dry residue was placed on an AND MS-70 thermal gravimetric humidity analyzer to determine the dry matter content in the hydrolysis mixture.

The liquid hydrolysis products were the raw materials for PHA production since they contain sugars which serve as a nutrient medium for prokaryotic microorganisms that synthesize this biopolymer.

The solid hydrolyzates, in turn, were dried and used as a filler to produce wood-filled biodegradable composites.

To produce the biopolymer, bacteria Cupriavidus necator was used, which can accumulate PHA in sufficient quantities. It is a common species that synthesizes P (3HB). The choice of this bacterium was determined by the fact that it provides one of the most effective fermentation process.

The bacteria were cultured in the medium of liquid wood hydrolyzates used as a carbon source, in feed batch fermentation, with sugar concentration maintained at a minimum of 10$20 \mathrm{~g} / \mathrm{l}$ for 50 hours of cultivation. The process was carried out on a rotary shaker (100 rpm) at $30{ }^{\circ} \mathrm{C}$ till the late stationary growth phase.

The cells and the supernatant fluid sediments were separated by centrifugation (60 min at $12000 \times \mathrm{g}$ ). Further, the separated cell sediment was dried for 24 hours at $80^{\circ} \mathrm{C}$ and stored at room temperature before use.

The solid wood hydrolysis product, lignin, was dried at $100^{\circ} \mathrm{C}$ in a laboratory oven and further additionally crushed.

To produce the composite samples, finely dispersed lignin and the resulting biodegradable polymer were mixed to a homogeneous polymer composition using a 
Brabender heated mixer. Further, an injection molding machine was used to make samples by injection molding.

Further tests of the composite samples were carried out on UGT-7045-MDL pendulum impact tester to determine the impact strength and on the UAI-7000 M testing machine to test the tensile strength.

\section{Results}

The results of experimental studies of the hydrolysis temperature impact on the total yield of aspen reducing substances showed that the optimal process mode was the temperature of $170^{\circ} \mathrm{C}$ (Fig. 2), which provides the highest yield of sugars after the hydrolysis.

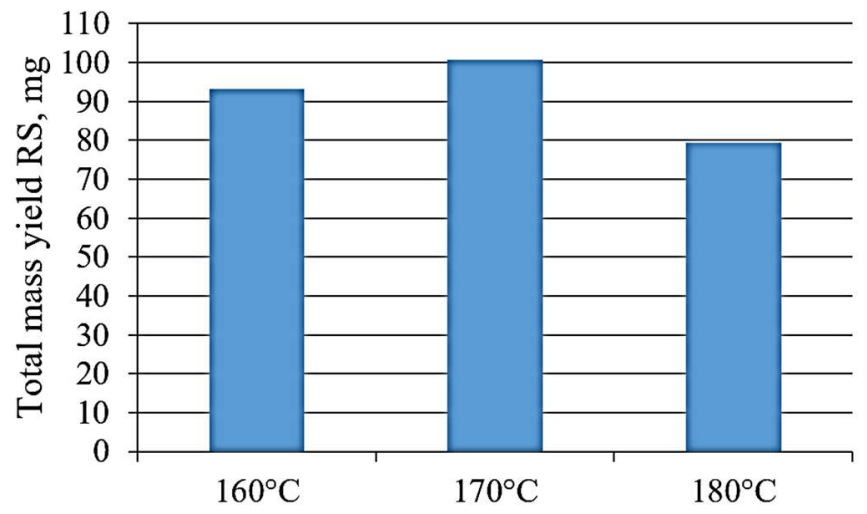

Fig. 2. Total RS mass yield depending on the hydrolysis temperature.

Next, the liquid hydrolysates were fed to a rotary shaker for cultivation of the Cupriavidus nicator strain in their medium. The microorganism's growth kinetics in liquid wood hydrolysates and PHA formation is shown in Fig. 3.

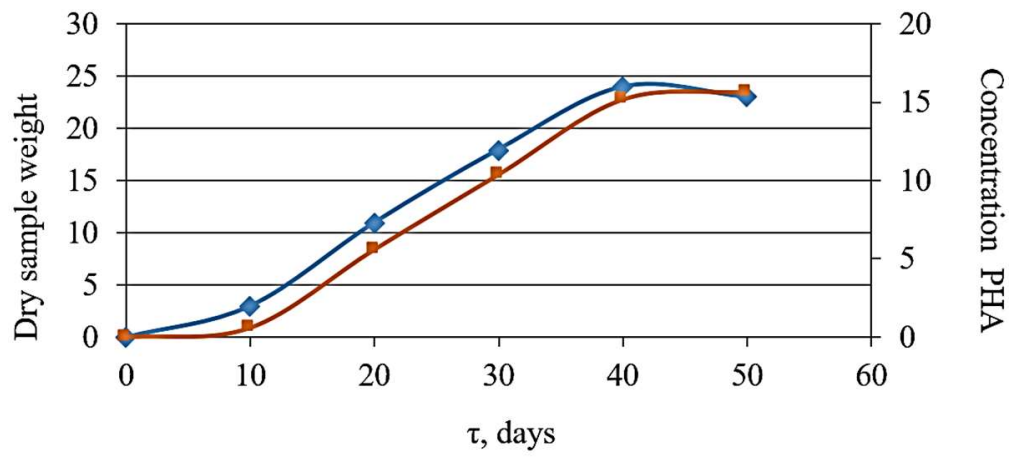

Fig. 3. Kinetics of microbial growth and PHA concentration increase in liquid wood hydrolyzates medium.

Fig. 3 shows that in the time interval from 20 to 40 days, both a sufficiently high rate of bacterial growth and a high rate of $\mathrm{P}(3 \mathrm{HB})$ synthesis occur. After 40 days of cultivation, the dry residue weight decreases, while the PHA yield continues to grow slightly, due to an increase in the concentration of the latter in the cell biomass.

Solid hydrolyzates were dried to a completely dry state and used as a filler in a mixture with a polymer obtained during cultivation by microorganisms. The studies of the mechanical characteristics of the resulting composite material are presented in Fig. 4-5. 
Fig. 4 shows the Charpy and Izod impact strength dependence diagrams. An increase in the amount of filler in the composite to $60 \%$ is accompanied with a decrease in both Izod and Charpy impact strength. Further, there is a slight increase in these values, which can be explained by an increase in the interaction of lignin particles with each other.

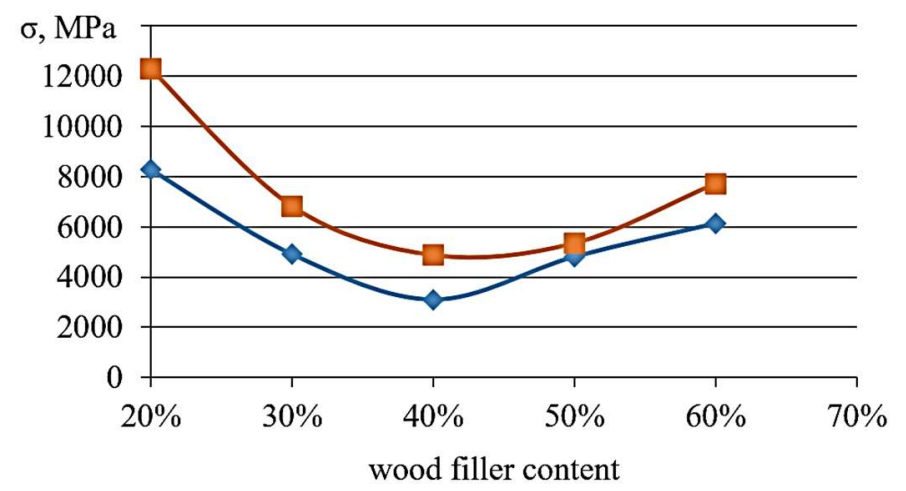

$\leadsto$ impact strength IZOD $-0-$ impact strenght CHARPY

Fig. 4. Charpy and Izod impact strength dependence diagrams.

Besides, based on the experiments, the tensile strength curves were plotted, which showed a decrease in this indicator with an increase in the filler content in the samples.

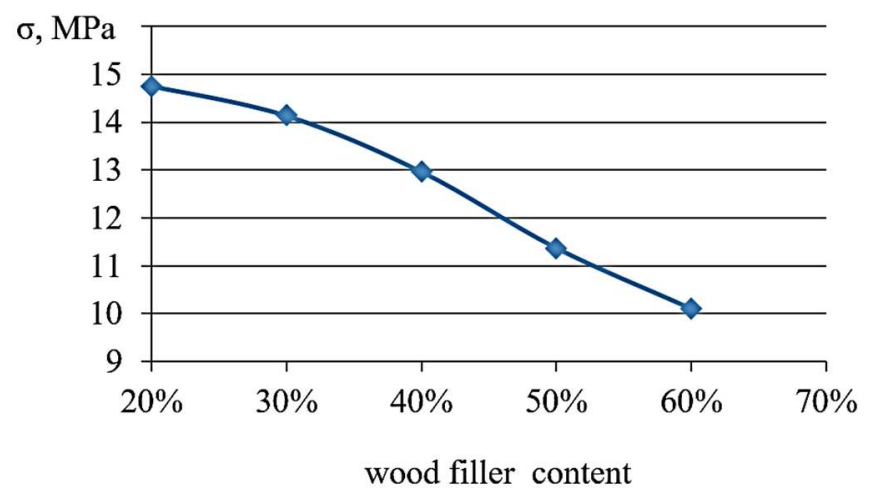

Fig. 5. Tensile strength with the trend line dependence diagram.

As can be seen from the curves in Figure 5, after the composite reaches a $40 \%$ filler content, the physical and mechanical characteristics of the composite significantly decrease.

\section{Discussion}

Based on the research, it was revealed that PHA production from wood involves pine sawdust hydrolysis in $4 \%$ sulfurous acid at $170^{\circ} \mathrm{C}$, followed by polymer matrix production from liquid hydrolyzates.

Mechanical studies of the properties of the composite material from a dried solid residue and a polymer matrix produced by microorganisms indicate that to ensure the possibility of creating building materials, the filler content should not exceed $40 \%$.

This composite can be used in finishing materials and biodegradable formwork production. 


\section{Conclusion}

The previous studies analysis indicates the undoubted prospects for the use of PHA as a polymer building material. At the same time, the extension of PHAs applications is constrained by their high cost, and therefore the search and attraction of affordable, cheap substrates is one of the key areas of biotechnology of these valuable macromolecules. It has been established that there are good reasons to use both the sugars released by hydrolysis and the solid residue.

The research allowed obtaining PHA-based biocomposites samples with a lignin filler, which were tested for strength. The curves showed the possibility of using wood filler in the production of cheap biodegradable composites for the construction industry. It has been found that in the range of the filler content within $40 \%$, the material will provide high strength characteristics, allowing creating finishing materials or biodegradable formwork from it.

\section{References}

1. G.A. Sabirova, N.R. Galavetdinov, R.R. Safin. Production of composite materials based on biodegradable components, Actual problems of the forest complex 55, 181-184 (2019). DOI: 10.1088/1742-6596/1399/4/044117.

2. A.Kh. Safiullina, R.R. Safin, Sh.R. Mukhametzyanov. Technology of pretreatment of wood filler with ozone in the production of composite materials, Actual problems of the forest complex 57, 60-63 (2020).

3. B. McAdam, M. Brennan Fournet, P. McDonald, M. Mojicevic. Production of Polyhydroxybutyrate (PHB) and Factors Impacting Its Chemical and Mechanical Characteristics, Polymers 12, 2908 (2020). DOI: 10.3390/polym12122908.

4. K. Shantini, A.R.M. Yahya, A.A. Amirul. Influence of Feeding and Controlled Dissolved Oxygen Level on the Production of Poly(3-Hydroxybutyrate-co-3-Hydroxyvalerate) Copolymer by Cupriavidus sp. USMAA2-4 and Its Characterization. Appl Biochem Biotechnol 176, 1315-1334 (2015). DOI: 10.1007/s12010-015-1648-5.

5. Evi Triwulandari, Muhammad Ghozali, Dewi Sondari, Melati Septiyanti, Yulianti Sampora, Yenny Meliana, Sri Fahmiati, Witta Kartika Restu, Agus Haryono. Effect of lignin on mechanical, biodegradability, morphology, and thermal properties of polypropylene/polylactic acid/lignin biocomposite, Plastics, Rubber and Composites 48:2, 82-92 (2019). DOI: 10.1080/14658011.2018.1562746.

6. Ariyanti Sarwono, Zakaria Man, M. Azmi Bustam, Duvvuri Subbarao, AlaminIdris, Nawshad Muhammad, Amir Sada Khan, Zahoor Ullah. Swelling mechanism of ureacrosslinked starch - lignin films in water, Environmental Technology 39:12, 1522-1532 (2018). DOI: 10.1080/09593330.2017.1332108.

7. Vebi Mimini, Eva Sykacek, Sharifah Nurul Ain Syed Hashim, Julian Holzweber, Hubert Hettegger, Karin Fackler, Antje Potthast, Norbert Mundigler, Thomas Rosenau. Compatibility of Kraft Lignin, Organosolv Lignin and Lignosulfonate With PLA in 3D Printing, Journalof Wood Chemistry and Technology 39:1, 14-30 (2019). DOI: 10.1080/02773813.2018.1488875.

8. Khaled F. El-Nemr, Hamdi R. Mohamed, Magdy A. Ali, Rasha M. Fathy, Abdelghaffar S. Dhmees. Polyvinyl alcohol/gelatin irradiated blends filled by lignin asgreen filler for antimicrobial packaging materials, International Journal of Environmental Analytical Chemistry 100:14, 1578-1602 (2020). DOI: 10.1080/03067319.2019.1657108.

9. O.A.T. Dias, D.R. Negrão, R.C. Silva, C.S. Funari, I. Cesarino, A.L. Leao. Studies of lignin as reinforcement for plastics composites, Molecular Crystals and Liquid Crystals 628:1, $72-78$ (2016). DOI: 10.1080/15421406.2015.1137677.

10. Rajendra Kumar Singla, Saurindra Nath Maiti, Anup Kumar Ghosh. Crystallization, Morphological, and Mechanical Response of Poly(Lactic Acid)/Lignin- 
BasedBiodegradable Composites, Polymer-Plastics Technology and Engineering 55:5, 475-485 (2016). DOI: 10.1080/03602559.2015.1098688.

11. Shaorong Lu, Shanrong Li, Jinhong Yu, Dong Guo, Rihua Ling, Bin Huang. The effect of hyperbranched polymer lubricant as a compatibilizer on the structure andproperties of lignin/polypropylene composites, Wood Material Science \& Engineering 8:3, 159-165 (2013). DOI: 10.1080/17480272.2013.769464.

12. J. Batog, R. Kozlowski, A. Przepiera. Lignocellulosic CompositesBonded by Enzymatic Oxidation of Lignin, Molecular Crystals and Liquid Crystals 484:1, 35-42 (2008). DOI: 10.1080/15421400801903387.

13. Beata Strzemiecka, Łukasz Klapiszewski, Danuta Matykiewicz, AdamVoelkel, Teofil Jesionowski. Functional lignin-SiO2 hybrids as potential fillers forphenolic binders, Journal of Adhesion Science and Technology 30:10, 1031-1048 (2016). DOI: 10.1080/01694243.2015.1115602.

14. Otávio Augusto Titton Dias, Djanira Rodrigues Negrão, Daniele Fernanda Chiarelli Gonçalves, Ivana Cesarino, Alcides Lopes Leão. Recent approaches and futuretrends for lignin-based materials, Molecular Crystals and Liquid Crystals 655:1, 204-223 (2017). DOI: 10.1080/15421406.2017.1360713.

15. Basheer Aaliya, Kappat Valiyapeediyekkal Sunooj, Maximilian Lackner. Biopolymer composites: a review, International Journal of Biobased Plastics 3:1, 40-84 (2021). DOI: 10.1080/24759651.2021.1881214.

16. H. Mohit, V. Arul Mozhi Selvan. A comprehensive review on surfacemodification, structure interface and bonding mechanism of plant cellulose fiber reinforced polymerbased composites, Composite Interfaces 25:5-7, 629-667 (2018). DOI: 10.1080/09276440.2018.1444832.

17. G.F. Ilalova, K.V. Saerova, R.R. Safin, Sh.R. Mukhametzyanov, A.H. Safiullina. Investigation of high-temperature hydrolysis of pine sawdust with sulfurous acid in order to increase the yield of reducing substance, Wood industry 3, 71-80 (2020).

18. K.V. Saerova, G.F. Ilalova, R.R. Safin, Sh.R. Mukhametzyanov, A.H. Safiullina. HighTemperature hydrolysis of pine sawdust with sulfurous acid to detect the concentration of reducing substances, Actual problems of the forest complex 57, 54-59 (2020).

19. Martin Boruvka, Lubos Behalek, Petr Lenfeld, Chakaphan Ngaowthong, Miroslava Pechociakova. Structure-related properties of bionanocomposites based onpoly(lactic acid), cellulose nanocrystals and organic impact modifier, Materials Technology 34:3, 143-156 (2019). DOI: 10.1080/10667857.2018.1540332.

20. G.A. Talipova, N.R. Galyavetdinov. Development of biodegradable composite materials made of polymer and vegetable filler, Actual problems of biology and ecology. Materials of the international scientific and practical conference, 235-240 (2019).

21. N.R. Galavetdinov, G.A. Sabirova, R.R. Safin, M.F. Galikhanov. Research of biodegradable wood-filled composite materials based on polylactide, Wood industry $\mathbf{3}$, 61-68 (2019).

22. Ali I., Jamil N. Enhanced biosynthesis of poly(3-hydroxybutyrate) from potato starch by Bacillus cereus strain 64-INS in a laboratory-scale fermenter, Prep Biochem Biotechnol 44 (8), 822-33 (2014). DOI: 10.1080/10826068.2013.867876.

23. M. Narodoslawsky, H.S.K. Shazad, R. Kollmann. LCA of PHA Production-Identifying the Ecological Potential of Bio-Plastic. Chem., Bio. Chem. Eng. Q. 29, 299-305 (2015). DOI: 10.15255/CABEQ.2014.2262.

24. N.R. Galyavetdinov, G.A. Talipova, R.R. Safin. Study of the Destructive Properties of Biodegradable Wood-Filled Composite Material, Materials Science Forum 992, 290-295 (2020). DOI: 10.4028/www.scientific.net/MSF.992.290.

25. S.R. Mukhametzyanov, R.R. Safin, N.R. Galyavetdinov. Improvement of Composite Filaments for Extrusive 3D Printing, Materials Science Forum 989, 827-832 (2020). DOI: 10.4028/www.scientific.net/MSF.989.827. 